\title{
On an Arithmetic Inequality
}

\author{
József Sándor
}

\begin{abstract}
We obtain an arithmetic proof and a refinement of the inequality $\varphi\left(n^{k}\right)+\sigma_{k}(n)<2 n^{k}$, where $n \geq 2$ and $k \geq 2$. An application to another inequality is also provided.
\end{abstract}

\section{$1 \quad$ Introduction}

If $n \geq 1$ is an integer, then let $\varphi(n)$ denote the classical Euler totient function, and $\sigma_{a}(n)$ be the sum of $a$ th powers of divisors of $n$ (with $a$ a real number).

Recently [2] H. Alzer and the author have shown that the divisibility

$$
n^{k} \mid\left(\varphi\left(n^{k}\right)+\sigma_{k}(n)\right)
$$

is not solvable for any integers $n \geq 2$ and $k \geq 2$. For $k=2$ this settled a conjecture of Adiga and Ramaswamy [1].

The proof of our result is based, besides arithmetical properties of $\varphi$ and $\sigma_{k}$, also on a Weierstrass product-type inequality, whose proof used methods of Mathematical analysis (as differentiability, and convex functions). In fact, the impossibility of (1) for $n \geq 2$ and $k \geq 2$, follows from the inequality

$$
\varphi\left(n^{k}\right)+\sigma_{k}(n)<2 n^{k}, n \geq 2, k \geq 2 .
$$

The aim of this note is to provide a completely arithmetic proof of inequality (2), and in fact to offer an improvement of this inequality.

Key Words: Divisibility, Euler totient, sum of divisors, number of divisors, Dedekind arithmetical function, inequalities.

2010 Mathematics Subject Classification: 11A25

Received: September 20, 2013.

Revised: November, 2013.

Accepted: November, 2013. 
We shall use also Dedekind's arithmetical function, defined by

$$
\psi(n)=n \prod_{p \mid n}(1+1 / p) \text { for } n \geq 2, \psi(1)=1 .
$$

It is clear that $\psi$, like $\varphi$ and $\sigma_{k}$, is a multiplicative function, i.e. satisfies $\psi(a b)=\psi(a) \psi(b)$ for $(a, b)=1$.

\section{Lemmas and Main Result}

In order to prove inequality (2) we need two auxiliary results.

The first lemma is stated in another form also in [2]; we present here its short proof for the sake of completeness.

Lemma 2.1. For all integers $n \geq 2$ and $k \geq 2$ we have

$$
\frac{\sigma_{k}(n)}{n^{k}} \leq \frac{\sigma_{2}(n)}{n^{2}}<\frac{n^{2}}{\varphi(n) \psi(n)}
$$

Proof. One has

$$
\sigma_{k}(n)=\sum_{d \mid n} d^{k}=\sum_{d \mid n}\left(\frac{n}{d}\right)^{k}=n^{k} \sum_{d \mid n} \frac{1}{d^{k}}
$$

which shows that $\frac{\sigma_{k}(n)}{n^{k}}$ is decreasing with respect to $k$. This leads to the first inequality of (3). Let now

$$
n=\prod_{j=1}^{r} p_{j}^{a_{j}} \geq 2
$$

be the prime factorization of $n$. Then

$$
\begin{gathered}
\frac{\sigma_{2}(n)}{n^{2}}=\prod_{j=1}^{r} \frac{p_{j}^{2 a_{j}+2}-1}{p_{j}^{2 a_{j}}\left(p_{j}^{2}-1\right)}=\prod_{j=1}^{r}\left(p_{j}^{2} \cdot \frac{1-1 / p_{j}^{2 a_{j}+2}}{p_{j}^{2}-1}\right) \\
<\prod_{p \mid n} \frac{p^{2}}{p^{2}-1}=\prod_{p \mid n} \frac{1}{\left(1-\frac{1}{p}\right)\left(1+\frac{1}{p}\right)}=\frac{n^{2}}{\varphi(n) \psi(n)} .
\end{gathered}
$$

This settles the second inequality in (3).

Lemma 2.2. For all $n \geq 1$ one has the inequality

$$
2 \frac{\psi(n)}{n} \geq 1+\frac{n}{\varphi(n)}
$$


Proof. Inequality (4) is stated without proof in [5]. Here we shall provide a complete proof.

It is easy to see that for $n=1$ and $n=p$ - prime, inequality (4) holds true; i.e. $2 \frac{p+1}{p} \geq 1+\frac{p}{p+1}$ is valid, with equality only for $p=2$. Since

$$
\frac{\psi\left(p^{a}\right)}{p^{a}}=\frac{\psi(p)}{p} \text { and } \frac{\varphi\left(p^{a}\right)}{p^{a}}=\frac{\varphi(p)}{p}
$$

for any primes $p$ and integers $a \geq 1$, clearly it is sufficient to prove (4) when $n$ is squarefree number, i.e. a product of distinct primes. Let us assume that $n$ is the least squarefree integer, for which (4) is false, and let $p$ be the greatest prime factor of $n$. Then $n$ can be written as $n=p \cdot m$, where $(p, m)=1$. Let $q$ denote the greatest prime factor of $m$. Then $q<p$. On the other hand, remark that

$$
\frac{m}{\varphi(m)}=\prod_{s \mid m, s \text { prime }} \frac{s}{s-1} \leq \frac{2}{1} \cdot \frac{3}{2} \cdot \frac{4}{3} \ldots \frac{s}{s-1} \ldots \frac{q}{q-1}=q,
$$

so

$$
\frac{m}{\varphi(m)} \leq q
$$

Now, by the definition of $n$ one has

$$
2 \frac{\psi(n)}{n}<1+\frac{n}{\varphi(n)}
$$

i.e.

$$
2 \frac{p+1}{p} \cdot \frac{\psi(m)}{m}<1+\frac{p}{p-1} \cdot \frac{m}{\varphi(m)} .
$$

Since $m<n$ and $m$ squarefree, by definition of $n$ one has

$$
2 \frac{\psi(m)}{m} \geq 1+\frac{m}{\varphi(m)} .
$$

Now multiplying both sides of (7) with $\frac{p+1}{p}$, and by taking into account of (6) we can write

$$
1+\frac{p}{p-1} \cdot \frac{m}{\varphi(m)}>\frac{p+1}{p}+\frac{p+1}{p} \cdot \frac{m}{\varphi(m)},
$$

i.e.

$$
\frac{1}{p(p-1)} \cdot \frac{m}{\varphi(m)}>\frac{1}{p} .
$$


From (8) we get

$$
p-1<\frac{m}{\varphi(m)} \leq q
$$

by relation (5). Since $q<p$, we get the contradiction $p-1<q<p$. This proves Lemma 2.2.

Theorem 2.1. For all $n \geq 2$ and $k \geq 2$ one has the inequality

$$
\frac{\varphi\left(n^{k}\right)}{n^{k}}+\frac{\sigma_{k}(n)}{n^{k}}<\frac{\varphi(n)}{n}+\frac{n^{2}}{\varphi(n) \psi(n)} \leq \phi+\frac{2}{1+\phi}<2,
$$

where $\phi=\frac{\varphi(n)}{n}<1$.

Proof. The first inequality of (8) follows by the remark that

$$
\varphi\left(n^{k}\right) / n^{k}=\varphi(n) / n,
$$

and by Lemma 2.1. For the second inequality use Lemma 2.2 in the form

$$
\frac{n}{\psi(n)} \leq \frac{2}{1+n / \varphi(n)}
$$

Finally, the last inequality is equivalent to

$$
\left(\frac{\varphi(n)}{n}\right)^{2}<\frac{\varphi(n)}{n}
$$

i.e. $\varphi(n)<n$, which is well-known. This concludes the proof of the theorem.

Remark 1. By the methods applied here, we have obtained a completely arithmetic study of problem (1) (see [2]).

An application. Let $d(n)$ denote the number of distinct divisors of $n$. The following theorem gives an improvement of a result from [3]:

Theorem 2.2. For all $n \geq 2$ not a prime number and $k \geq 2$ one has the inequalities

$$
\frac{\sigma_{k}(n)}{n^{k}}<\frac{2 n}{n+\varphi(n)}<\frac{d(n)}{2} .
$$

Proof. The first inequality follows by a combination of relations (3) and (10). As the second inequality may be written as $n d(n)+d(n) \varphi(n)>4 n$, remark that this is true for $d(n) \geq 3$, since by a well known inequality of R. Sivaramakrishnan [4] one has $d(n) \varphi(n)>n$ for all $n>1$. Clearly, $d(n)=2$ only if $n$ is a prime, so the result follows. 
Remark 2. The weaker inequality of (11), in case when $n$ has at least two distinct prime factors, appears in paper [3], as a corollary to more general results.

Acknowledgements. The author thanks Professor H. Alzer for discussions on this and related topics.

The publication of this paper is supported by the Grant of Romanian National Authority for Scientic Research CNCS-UEFISCDI, Project No. PN II-ID-WE-2012-4-161

\section{References}

[1] C. Adiga, H.N. Ramaswamy, A note on certain divisibility problem, Int. J. Math. Anal., 2(2008), 1157-1161.

[2] H. Alzer, J. Sándor, On a divisibility problem, Rend. Sem. Mat. Univ. Padova, vol. 130(2013), 215-220.

[3] J. Sándor, An application of the Jensen-Hadamard inequality, Nieuw Arch.Wiskunde (4) 8(1990), 63-66

[4] R. Sivaramakrishnan, Problem E 1962., Amer.Math.Monthly, 74(1967), p.198

[5] Ch. R. Wall, Density bounds for Euler's function, Math. Comp., 26(1972), 779-783.

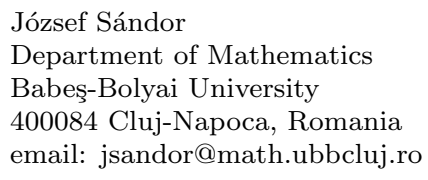

